\title{
вмJ Global Health COVID-19 and non-communicable diseases in the Eastern Mediterranean Region: the need for a syndemics approach to data reporting and healthcare delivery
}

\author{
Ghiwa Nassereddine, ${ }^{1}$ Samia Habli, ${ }^{1}$ Slim Slama, ${ }^{2}$ Kasturi Sen, ${ }^{3}$ Anthony Rizk, ${ }^{4}$ \\ Abla M Sibai ${ }^{1}$
}

\begin{abstract}
To cite: Nassereddine G, Habli S Slama S, et al. COVID-19 and non-communicable diseases in the Eastern Mediterranean Region: the need for a syndemics approach to data reporting and healthcare delivery. BMJ Global Health 2021;6:e006189. doi:10.1136/ bmjgh-2021-006189
\end{abstract}

GN and SH contributed equally.

Received 3 May 2021 Accepted 18 May 2021

Check for updates

(C) Author(s) (or their employer(s)) 2021. Re-use permitted under CC BY-NC. No commercial re-use. See rights and permissions. Published by BMJ.

${ }^{1}$ Faculty of Health Sciences, American University of Beirut, Beirut, Lebanon

${ }^{2}$ WHO Regional Office for the Eastern Mediterranean, Cairo, Egypt

${ }^{3}$ Wolfson College (CR), University of Oxford, Oxford, UK ${ }^{4}$ Department of Anthropology and Sociology, Graduate Institute of International and Development Studies, Geneva, Switzerland

\section{Correspondence to} Anthony Rizk; anthony.rizk@graduateinstitute. ch
As of 30 April 2021, the Eastern Mediterranean Region (EMR), home to nearly 700 million people across 22 diverse countries, has reported 9109162 confirmed cases of COVID-19 and 182654 cumulative deaths since the beginning of the pandemic. ${ }^{1}$ Pandemic response in the EMR is complicated by compromised healthcare systems, prolonged conflicts and humanitarian crisis, and suboptimal reporting and transparency in many countries of the region. ${ }^{2}$ Consequently, COVID-19 incidence rates have been uneven, fluctuating from less than 1000 confirmed cases per million population in Sudan, Syria, Somalia and Yemen to more than 40000 confirmed cases per million population in Lebanon, Qatar and Bahrain. Up to this point in the pandemic, the five countries hardest hit with cumulative COVID-19 deaths per capita have been Lebanon, Iran, Tunisia, Palestine and Jordan and with the highest overall cumulative deaths have been Iran, Iraq, Pakistan, Egypt and Morocco. ${ }^{3}$

In tandem, as the COVID-19 pandemic disrupts the delivery of health services, the EMR has been reported to have the highest average disruption with $75 \%$ of essential health services (EHS) being compromised in 13 countries. ${ }^{4}$ Existing healthcare infrastructures in the EMR have been repurposed to respond to the pandemic, with many of the disruptions affecting services designed for the management, treatment and care of noncommunicable diseases (NCDs). More than $40 \%$ of countries reported partial or full disruption to hypertension and diabetes management, cancer treatment, asthma services, rehabilitation services and palliative care. This is despite official reporting that continuity of NCD services is included as essential services in national COVID-19 strategic preparedness

\section{Summary box}

As elsewhere, health services designed for the management and treatment of non-communicable diseases (NCDs) have been repurposed to respond to COVID-19 in many countries of the Eastern Mediterranean Region (EMR).

- Many countries of the EMR are distinctly challenged by compromised healthcare systems, prolonged political unrest, conflict and humanitarian crisis, poor vital registration systems, suboptimal reporting and dearth in publicly available data.

- COVID-19 and NCDs are a syndemic-their synergistic relationship leaves people living with NCDs at a higher risk of developing severe COVID-19 complications, and patients who recover from COVID-19 are more likely to develop long-term chronic conditions.

- In the EMR, it is now more imperative than ever to recognise the interconnectedness of communicable diseases and NCDs, build stronger public health systems to achieve universal health coverage and establish reliable surveillance systems.

- Working long-term and recognising how COVID-19 and NCDs are syndemically interlocked conditions may be the first step towards developing the nuanced approaches that are needed to more comprehensively protect society's vulnerable populations.

and response plans. ${ }^{5}$ The United Nations Development Programme's NCD COVID-19 Vulnerability Index-designed to assess countries' COVID-19 vulnerability due to the underlying prevalence of NCDs and NCD risk factors-reports that 15 of 22 countries in the EMR rank above the global average. Even as vaccines for COVID-19 roll out globally, inoculating the vast majority of the world's population will be a global health imperative for some time to come, and a short-term end to the pandemic remains out of reach. In the interim, priorities and practices left 
unchanged, NCD care will continue to suffer in the region and beyond. ${ }^{6}$

In recent commentaries, ${ }^{78}$ COVID-19 and NCDs have increasingly been referred to as a syndemic-defined by Singer et al as socially determined and co-occurring diseases that interact to produce disease clusters. ${ }^{9}$ The synergism between COVID-19 and NCDs is doublesided. ${ }^{10}$ On the one hand, existing NCDs, such as cardiovascular disease, diabetes mellitus, cancer and chronic obstructive pulmonary disease, have been shown to cause higher risk of complications, higher likelihood of need of intensive care unit admissions, and poorer prognosis and increased mortality among patients with COVID-19. ${ }^{11}$ On the other hand, patients who survive COVID-19 are likely to develop or have further entrenched long-term chronic conditions. $^{12}$

More fundamentally, a syndemics approach emphasises how disease clusters are socially, economically and politically determined. As Maani et al note, health and wellness compromised by social inequalities in prepandemic times became and will continue to be reflected in poor health outcomes associated with NCDs throughout the course of the pandemic going forward. ${ }^{13}$ Social determinants of NCDs, such as loss of livelihood, poor nutrition and psychological distress, will only continue to increase with growing global inequalities and poverty, which are further exacerbated by the economic impact of COVID-19 pandemic control measures. ${ }^{13}$ Mendenhall ${ }^{14}$ emphasises that context matters in syndemics. In some places, the pandemic has exposed the fragility of NCD care and weaknesses in the way health systems are funded and organised. ${ }^{14}$ This is particularly important for forcibly displaced persons and refugees and for resource-scarce communities and reflects, in turn, the extreme impact of poverty, on the one hand, and privilege, on the other, in determining exposure and shielding from transmission. The EMR is such a context. To take one example among many, mortality among Palestinian refugees in Lebanon has been reported to be three times higher than the Lebanese population, a finding attributed to pre-existing conditions such as pulmonary and cardiac diseases, all against a background of poverty and overcrowding. ${ }^{15}$

These factors will become even more challenging in the future as health systems in many countries run out of funding for the spillover effect of the NCD-COVID-19 syndemic on public health. Although the global vaccination programme for COVID-19 is underway, the pursuit of a solely biomedical response to this syndemic will continue to fall short of expectations so long as it does not address the social and economic determinants of disease and the nuanced interactions between COVID-19 and NCDs. Despite this, disproportionately less attention has been paid in the global pandemic response to the challenges that NCDs pose. This has included, and not been exceptional to, the EMR.

Even before the COVID-19 pandemic, reduction in premature NCD mortality rates experienced a global slowdown in the past decade. ${ }^{16}$ During the pandemic response, funding and human resources have further shifted to cater specifically for the emergency, prioritising care for patients with COVID-19 over many other diseases, including NCDs. ${ }^{12}$ Hospital and clinic staff, including physicians from specialties that are not related to infectious diseases, as well as epidemiologists and nurses, have been redeployed to serve in emergency departments and intensive care to care for patients with COVID-19. ${ }^{12}$ Furthermore, lockdown measures in most countries have disrupted continuity of care for patients with NCDs and postponement of routing medical appointments and tests affecting healthcare service access and availability to people with NCDs. ${ }^{5}$ Restrictions to mobility due to lockdown measures have limited access to preventive and control services. ${ }^{12}$ This has resulted in diminishing returns of earlier investments towards achieving Sustainable Development Goals (SDGs) in reducing premature mortality from NCDs by one-third by $2030 .{ }^{17}$ Such a 'covidisation' of care will likely lead to unintended long-term consequences, even as the COVID-19 pandemic subsides.

The pandemic has increased our awareness of the several endemic concerns to the EMR. It has also emphasised key areas for reform, the resolution of which is long overdue.

First, it is now more imperative than ever before to build stronger public health systems to achieve universal health coverage (UHC) and ensure all people are able to receive the spectrum of health services they need-promotion, prevention, treatment, rehabilitation and palliation. ${ }^{18}$ The COVID-19 pandemic is a striking reminder of the interconnectedness of UHC and health emergencies, of the need to invest more towards achieving a target $3 \cdot 8$ of the United Nations SDGs where 'no one is left behind'.

Second, the lack of reliable surveillance system for monitoring and control and the dearth in publicly available data have made building data infrastructures by regional governments vital going forward. ${ }^{19}$ Many health information systems are currently weak and do not capture NCD service utilisation and outcomes. ${ }^{20}$ It is impossible to appropriately plan disease outbreak response strategies without reliable, disaggregated, transparent and openly accessible data, and an extensive health database to track baseline measures of NCD burdens integrated into larger surveillance systems needs to be escalated to a regional strategic priority. ${ }^{18}{ }^{21}$ Even where data are available, it is not often sufficiently used to inform policy responses to the pandemic. As recommended in WHO guidance on maintaining EHS, countries should be able to identify key EHS and strengthen their monitoring. ${ }^{22}$

Third, more accurate attribution of the contributory causes to the underlying cause of death is urgently needed to allow reporting of data that would substantiate the interconnectedness of COVID-19 and NCDs. At the moment, deaths due to NCDs are often miscounted as caused by COVID-19 only, furthering the deprioritisation of NCDs on the global agenda. Adopting a syndemics approach to cause of death structure will require that both COVID-19 and NCDs contributing to death are 
acknowledged in reporting practices to identify causes of excess deaths as being because of or with COVID-19. ${ }^{23}$

Fourth, for many patients recovered from COVID-19 worldwide, SARS-CoV-2 may be becoming the source of emerging chronic diseases in the near future, further bolstering the burden of NCD management and care. In this new normal, the dichotomy between communicable diseases and NCDs may be fraying. ${ }^{10}$ The EMR, and the world more broadly, needs to recognise how disease outbreaks are interlocked and syndemic, and plan appropriately going forward, such as with emergency and disaster reduction policies.

The COVID-19 pandemic has exposed how deeply linked NCDs and communicable diseases can be and the perils of continuing to treat these issues in siloes. Working towards the long-term and recognising how COVID-19 and NCDs are syndemically interlocked diseases may be the first step towards developing the nuanced approaches that are needed to more comprehensively protect, rather than further compromise, society's vulnerable populations. Adopting a syndemics approach to COVID-19 and NCDs allows us to recognise how political and social factors drive, perpetuate or worsen the emergence and clustering of diseases. As such, there is an urgent need to embrace renewed attention to NCD prevention and management in national responses to COVID-19. The ongoing COVID-19 pandemic can, and must, be leveraged towards enhanced opportunities for NCD care. Given the global reach of covidisation of care, we urge global health actors as well as regional policy-makers in the EMR to chart a way forward that develops a comprehensive response appropriate to the complexity of the problem. As syndemic diseases, COVID-19 and NCDs are acting in concert; healthcare responses need to adapt to this new reality.

Twitter Abla M Sibai @abla_sibai

Contributors GN, SH, AR and AMS prepared the first draft of the manuscript. SS and KS revised the manuscript in its different stages. AMS and AR led the outline of the manuscript. All authors read and revised the final version of the manuscript and approved it for submission.

Funding This work was supported with funding to the senior author, AMS, from the Global Challenges Research Fund through UKRI/ESRC for the RECAP Project (ES/P010873/1).

Competing interests None declared.

Patient consent for publication Not required.

Provenance and peer review Not commissioned; internally peer reviewed.

Data availability statement There are no data in this work.

Open access This is an open access article distributed in accordance with the Creative Commons Attribution Non Commercial (CC BY-NC 4.0) license, which permits others to distribute, remix, adapt, build upon this work non-commercially, and license their derivative works on different terms, provided the original work is properly cited, appropriate credit is given, any changes made indicated, and the use is non-commercial. See: http://creativecommons.org/licenses/by-nc/4.0/.

\section{REFERENCES}

1 WHO. WHO coronavirus disease (COVID-19) Dashboard, 2021. Available: https://covid19. who.int [Accessed $30 \mathrm{Apr}$ 2021].

2 Baloch Z, Ma Z, Ji Y, et al. Unique challenges to control the spread of COVID-19 in the middle East. $J$ Infect Public Health 2020;13:1247-50.

3 EMRO WHO. Eastern Mediterranean regional office COVID-19 Dashboard, 2021. Available: https://app.powerbi.com/view?r=eyJr ljoiN2ExNWI3ZGQtZDk3My00YzE2LWFjYmQtNGMwZjk0OWQ1 MjFhliwidCI6ImY2MTBjMGI3LWJkMjQtNGlzOS04MTBiLTNkYzl4 MGFmYjU5MClsImMiOjh9 [Accessed 12 Feb 2021].

4 Al-Mandhari AS, Brennan RJ, Abubakar A, et al. Tackling COVID-19 in the Eastern Mediterranean Region. The Lancet 2020;396:1786-8.

5 EMRO WHO. Rapid assessment of service delivery for NCDs during COVID-19, 2020. Available: http://www.emro.who.int/ noncommunicable-diseases/publications/rapid-assessment-ofservice-delivery-for-ncds-during-covid-19.html?ver=2 [Accessed 12 Feb 2021].

6 UNDP. NCD COVID-19 vulnerability Dashboard (Beta), 2021. Available: https://public.domo.com/embed/pages/aOV4p [Accessed 12 Feb 2021].

7 Horton R. Offline: COVID-19 is not a pandemic. Lancet 2020;396:874.

8 Yadav UN, Rayamajhee B, Mistry SK, et al. A Syndemic perspective on the management of non-communicable diseases amid the COVID-19 pandemic in low- and middle-income countries. Front Public Health 2020;8:508.

9 Singer M, Bulled N, Ostrach B, et al. Syndemics and the biosocial conception of health. Lancet 2017;389:941-50.

10 Sheldon TA, Wright J. Twin epidemics of covid-19 and noncommunicable disease. BMJ 2020;369:m2618.

11 Pal R, Bhadada SK. COVID-19 and non-communicable diseases. Postgrad Med J 2020;96:429-30.

12 Palmer K, Monaco A, Kivipelto M, et al. The potential longterm impact of the COVID-19 outbreak on patients with noncommunicable diseases in Europe: consequences for healthy ageing. Aging Clin Exp Res 2020;32:1189-94.

13 Maani N, Abdalla SM, Galea S. Avoiding a legacy of unequal non-communicable disease burden after the COVID-19 pandemic. Lancet Diabetes Endocrinol 2021;9:133-5.

14 Mendenhall E. The COVID-19 syndemic is not global: context matters. The Lancet 1731;2020:396.

15 Azhari T. Palestinian refugees in Lebanon three times more likely to die with COVID-19. Reuters, 2021. Available: https://www.reuters. com/article/us-lebanon-refugees-health-trfn-idUSKBN2AG22M [Accessed 21 Feb 2021].

16 Martinez R, Lloyd-Sherlock P, Soliz P, et al. Trends in premature avertable mortality from non-communicable diseases for 195 countries and territories, 1990-2017: a population-based study. Lancet Glob Health 2020;8:e511-23.

17 Bergman M, Jagannathan R, Narayan KMV. Nexus of COVID-19 and diabetes pandemics: global public health lessons. Diabetes Res Clin Pract 2020;164:108215.

18 Jabbour M, Hemadi N, Abou Samra C. Universal health coverage in times of COVID-19. Pandemic: Actions for EMR Countries, 2020.

19 Wehbe S, Fahme SA, Rizk A, et al. COVID-19 in the middle East and North Africa region: an urgent call for reliable, disaggregated and openly shared data. BMJ Glob Health 2021;6:e005175.

20 Gouda HN, Richards NC, Beaglehole R, et al. Health information priorities for more effective implementation and monitoring of non-communicable disease programs in low- and middle-income countries: lessons from the Pacific. BMC Med 2015;13:233.

21 Slama S, Kim H-J, Roglic G, et al. Care of non-communicable diseases in emergencies. Lancet 2017;389:326-30.

22 WHO. World health statistics 2020 visual summary, 2020. Available: https://www.who.int/data/gho/whs-2020-visual-summary [Accessed $12 \mathrm{Feb} 2021]$

23 Vandoros S. Excess mortality during the Covid-19 pandemic: early evidence from England and Wales. Soc Sci Med 2020;258:113101. 